\title{
Partial Response to First-Line Crizotinib in an Elderly Male Patient with ROS1 Translocation-Positive Lung Cancer
}

\author{
Tobias R. Overbeck ${ }^{a, b} \quad$ Katja Schmitz ${ }^{c} \quad$ Christoph Engelke ${ }^{d}$ \\ Carsten-Oliver Sahlmann ${ }^{\mathrm{e}}$ Sara Hugo $^{c}$ Laura Kellner ${ }^{c}$ \\ Lorenz Trümper ${ }^{a, b}$ Hans-Ulrich Schildhaus ${ }^{a, c}$ \\ ${ }^{a}$ Lungentumorzentrum Universität Göttingen and Göttingen Comprehensive Cancer \\ Center (G-CCC), ${ }^{b}$ Department of Hematology and Medical Oncology, ${ }^{c}$ Institute of \\ Pathology, ${ }^{\mathrm{d}}$ Department of Radiology, and ${ }^{\mathrm{e}}$ Department of Nuclear Medicine, \\ University Hospital Göttingen, Göttingen, Germany
}

\section{Key Words}

Precision medicine $\cdot$ NSCLC $\cdot$ ROS1 rearrangement $\cdot$ Fluorescence in situ hybridization

\begin{abstract}
We report on a 90-year-old male patient with a ROS1-translocated adenocarcinoma of the lung who was treated with crizotinib as first-line therapy. After 11 months of treatment, we noticed complete metabolic response as measured by ${ }^{18}$ F-FDG-PET/CT scan and a partial response according to RECIST criteria. This patient indicates that ROS1 translocations are not restricted to young age, female gender and low stage. Furthermore, this case illustrates exemplarily that crizotinib therapy is effective and manageable even as first-line treatment in elderly patients with comorbidities. Based on our findings, we recommend to include elderly patients with advanced pulmonary adenocarcinomas in molecular screening approaches for ROS1 translocations.

\section{Introduction}

Personalized treatment in the context of precision medicine has been shown to be effective in the therapy of lung cancer patients [1]. Activating rearrangement of the receptor ty-

\section{KARGER}

Prof. Hans-Ulrich Schildhaus, MD

Institute of Pathology

University Hospital Göttingen

Robert-Koch-Strasse 40, DE-37075 Göttingen (Germany)

E-Mail hans-ulrich.schildhaus@med.uni-goettingen.de 
Overbeck et al.: Partial Response to First-Line Crizotinib in an Elderly Male Patient with ROS1 Translocation-Positive Lung Cancer

rosine kinase ROS1 represents one molecular subtype of pulmonary adenocarcinomas [2] which can be inhibited by targeted treatment. Recently, it has been demonstrated that crizotinib, a small molecule which is approved for treatment of $A L K$-positive lung cancer, has additional activity in pulmonary carcinomas with ROS1 translocations [3, 4]. Therefore, molecular screening for ROS1 rearrangements is likely to become implemented in most clinical settings. In the context of screening approaches, it is noteworthy that data from reported cohorts indicate that patients with ROS1-positive cancers are considerably younger than ROS1-negative ones [4-6]. In a recent report including 72\% non-Asian individuals with ROS1 translocation, the median age at onset was only 49.8 years which was significantly lower than in ROS1-negative patients (mean age: 62.3 years) and even lower than in $A L K$ translocated carcinomas (mean age: 51.6 years) [7]. This is why it is suspected that molecular screening for ROS1 translocations could be restricted to younger patients. For elderly individuals, however, there is a great clinical need for tailored therapeutics. Especially effective first-line treatments quickly restoring quality of life are needed since ageing patients suffer frequently from comorbidities which do not allow conventional therapies.

\section{Case Report}

A 90-year-old male patient presented with dyspnea to our department. His medical history included coronary heart disease with myocardial infarction and double coronary stent implantation 4 months before as well as a negative smoking history. Further relevant comorbidities were pericarditis epistenocardica and chronic renal failure. CT scans revealed a tumor mass of 33-mm maximum size in the lower left lobe of the lung and a supposed me-

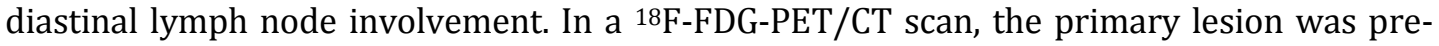
sent with a SUVmax of 19.7 (fig. 1). Furthermore, PET/CT-positive hilar and mediastinal lymph nodes were found at positions $11 \mathrm{~L}$ and 5 . These findings resulted in a UICC stage IIIA (cT2a, cN2, cM0). The mediastinal lymph node was not reached by endobronchial workup.

A core needle biopsy was taken. At histological examination, a poorly differentiated adenocarcinoma of predominant solid subtype was found. Immunohistochemistries for cytokeratin 7 and thyroid transcription factor-1 were positive, whereas negative immunostains for p63 and CD56 ruled out squamous cell and neuroendocrine carcinoma subtypes. Molecular tests for EGFR and KRAS mutations as well as for $A L K$ translocation and MET amplification were all negative. However, fluorescence in situ hybridization (FISH) analysis for ROS1 rearrangement showed aberrant probe signals in 81 of 100 evaluated tumor cell nuclei (fig. 2). Therefore, the diagnosis of a poorly differentiated pulmonary adenocarcinoma with ROS1 translocation could be established.

Due to tumor stage, comorbidity and age the patient was not eligible for surgery or conventional chemotherapy. Stereotactic radiotherapy of the primary lesion was discussed but not favored due to the molecular finding of a ROS1 translocation. After tumor board discussion and informed consent, the patient was treated with crizotinib at a daily dose of initially twice $250 \mathrm{mg}$ p.o. Lab results at day 7 showed a further increase of already previously elevated gamma-glutamyl transpeptidase (GGT) from twice to four times of the upper normal limit (UNL) and an elevation of glutamate-oxaloacetate transaminase (GOT) and aspartateamino transferase (ASAT) from normal to twice of UNL. In the same period, serum creatinine raised from 1.6 to $2.0 \mathrm{mg} / \mathrm{dl}$. Following 7 days of drug withdrawal, crizotinib was restarted with a reduced daily dose of $250 \mathrm{mg}$, while lab parameters had improved with persistently elevated serum creatinine at the former level. The patient reported discomfort consisting of nausea, emesis and diarrhea, starting at day 5 after reduction of the dose level. On day 15 of 
Overbeck et al:: Partial Response to First-Line Crizotinib in an Elderly Male Patient with ROS1 Translocation-Positive Lung Cancer

crizotinib $250 \mathrm{mg}$ p.o. once daily, the patient suffered from thoracic pain, and he stopped medication. With a delay of 3.5 weeks, the patient restarted therapy with crizotinib $250 \mathrm{mg}$ once daily again. An acute ulcerative fibrinous purulent esophagitis (month 4.5) resolved with a H2-blocking oral therapy. In summary, over a period of 11 months, adverse events caused a cumulative off-drug interval of 11.5 weeks. The first tumor evaluation was done by chest X-ray 2 and 3 months after initiation of crizotinib. Planar measures of the primary lesion decreased from $26 \times 50 \mathrm{~mm}$ at baseline to $18 \times 42$ and $13 \times 34 \mathrm{~mm}$, respectively. CT scans after 4.5 months revealed that the primary lesion showed a partial remission from 33 to $13 \mathrm{~mm}$ (CT) and complete metabolic response (PET). At this time, the hilar and mediastinal lymph nodes were still present in CT and PET scans. X-ray of the chest at months 7 and 10 showed no relevant changes. After 11 months, the patient is still under treatment (crizotinib $250 \mathrm{mg}$ daily, half of the recommended dose) without any limitation in daily living and a performance status of ECOG 0 .

\section{Discussion}

ROS1 translocations occur in $1.8 \%$ of pulmonary adenocarcinomas regardless of tumor stage and grading (personal observation). This frequency will result in an estimated total number of 2,500-3,300 ROS1-positive lung cancers in the US in 2015. Very recent data have provided evidence that crizotinib, a TKI with anti-MET, anti-ALK, anti-RON and anti-ROS1 activity, is highly active in patients with ROS1-translocated lung cancers [3, 4]. This treatment has been shown to result in durable, long-lasting tumor shrinkage and good clinical symptom control.

All previous clinical reports on ROS1 alterations in lung cancer have, however, emphasized that ROS1-associated adenocarcinomas are merely restricted to lower clinical stage, female gender and to younger patients. ROS1 translocations have been reported in an age range from 25 to 77 years, and the mean age was even lower than in ALK-positive lung cancers $[4,7]$.

In this report, we describe an elderly male patient with an advanced ROS1 positive pulmonary adenocarcinoma who experienced metabolic response and significant tumor shrinkage under TKI treatment. A partial response according to RECIST 1.1 criteria was achieved within 4.5 months. Due to co-morbidity our patient was not eligible to standard therapy regimens, a fact which occurs frequently in elderly individuals. Compared to conventional chemo- or radio-chemotherapy the safety profile of crizotinib is suitable for many patients with co-morbidities, and severe adverse effects have been only rarely described [4, 8]. In our case, crizotinib therapy was clinically manageable. Elevation of transaminases and clinical discomfort required pausing of drug administration and dose reduction. Clinical response was, however, achieved even at $250 \mathrm{mg}$ crizotinib daily. Most importantly, overall quality of life could quickly be restored, and throughout the entire therapy, the patient could be prevented from hospital admission and toxic chemotherapy.

Since we anticipated good response to TKI therapy, we decided to treat our patient with crizotinib directly as first-line therapy. Up to now there are, however, only limited data on this drug as a first-line treatment in ROS1-translocated tumors. To the best of our knowledge, only 7 patients have been reported so far [4] who all responded to that treatment. These data and our findings might provide a rationale for first-line crizotinib treatment of ROS1-positive tumors in clinical trials.

Our case demonstrates that fast clinical decisions on therapeutic options require fast provision of results from molecular screening. Therefore, we recommend applying molecu- 
Overbeck et al.: Partial Response to First-Line Crizotinib in an Elderly Male Patient with ROS1 Translocation-Positive Lung Cancer

lar panels as reflex test. For the detection of ROS1 translocations, FISH appears to be best suitable since this method provides data within only 2 days after initial diagnosis. It has been shown that there is no correlation between the type of translocation, i.e., the specific translocation partner of ROS1, and response to crizotinib [4]. Since FISH is an assay which detects all subtypes of ROS1 rearrangements, this technique again is currently the most appropriate method for detection of ROS1 alterations.

In conclusion, we report here the so far oldest patient with a ROS1-positive lung cancer, indicating that ROS1 translocations are not restricted to young age, female gender and low stage. This case illustrates exemplarily that crizotinib therapy is effective and manageable even as first-line treatment in elderly patients with comorbidities. Based on our findings, we recommend to include elderly patients with advanced pulmonary adenocarcinomas in molecular screening approaches for ROS1 translocations.

\section{Acknowledgement}

The authors are grateful to our patient who agreed with this report.

\section{Statement of Ethics}

Publication was permitted by the patient.

\section{Disclosure Statement}

H.U.S. has received research grants by Novartis Oncology and ZytoVision (outside of this work), and honoraria and reimbursements for advisory membership and lectures by Pfizer, Novartis, Roche, Zytomed, ZytoVision and Abbott Molecular. T.O. has received honoraria and reimbursements for advisory membership and lectures by Astra Zeneca, BMS, Boehringer Ingelheim, Clovis Oncology, Lilly, Medac, MSD, Novartis, Sanofi-Aventis and Roche.

\section{References}

1 Clinical Lung Cancer Genome Project (CLCGP); Network Genomic Medicine (NGM): A genomics-based classification of human lung tumors. Sci Transl Med 2013;5:209ra153.

-2 Rikova K, Guo A, Zeng Q, et al: Global survey of phosphotyrosine signaling identifies oncogenic kinases in lung cancer. Cell 2007;131:1190-1203.

-3 Bos M, Gardizi M, Schildhaus HU, et al: Complete metabolic response in a patient with repeatedly relapsed non-small cell lung cancer harboring ROS1 gene rearrangement after treatment with crizotinib. Lung Cancer 2013;81:142-143.

4 Shaw AT, Ou S-HI, Bang Y-J, et al: Crizotinib in ROS1-rearranged non-small-cell lung cancer. New Engl J Med 2014;371:1963-1971.

5 Chen Y-F, Hsieh M-S, Wu S-G, et al: Clinical and the prognostic characteristics of lung adenocarcinoma patients with ROS1 fusion in comparison with other driver mutations in East Asian populations. J Thorac Oncol 2014;9:1171-1179.

-6 Yoshida A, Kohno T, Tsuta K, et al: ROS1-rearranged lung cancer: a clinicopathologic and molecular study of 15 surgical cases. Am J Surg Pathol 2013;37:554-562.

7 Bergethon K, Shaw AT, Ignatius Ou S-H, et al: ROS1 rearrangements define a unique molecular class of lung cancers. J Clin Oncol 2012;30:863-870.

-8 Ono A, Takahashi T, Oishi T, et al: Acute lung injury with alveolar hemorrhage as adverse drug reaction related to crizotinib. J Clin Oncol 2013;31:e417-e419. 


\section{Case Reports in Oncology}
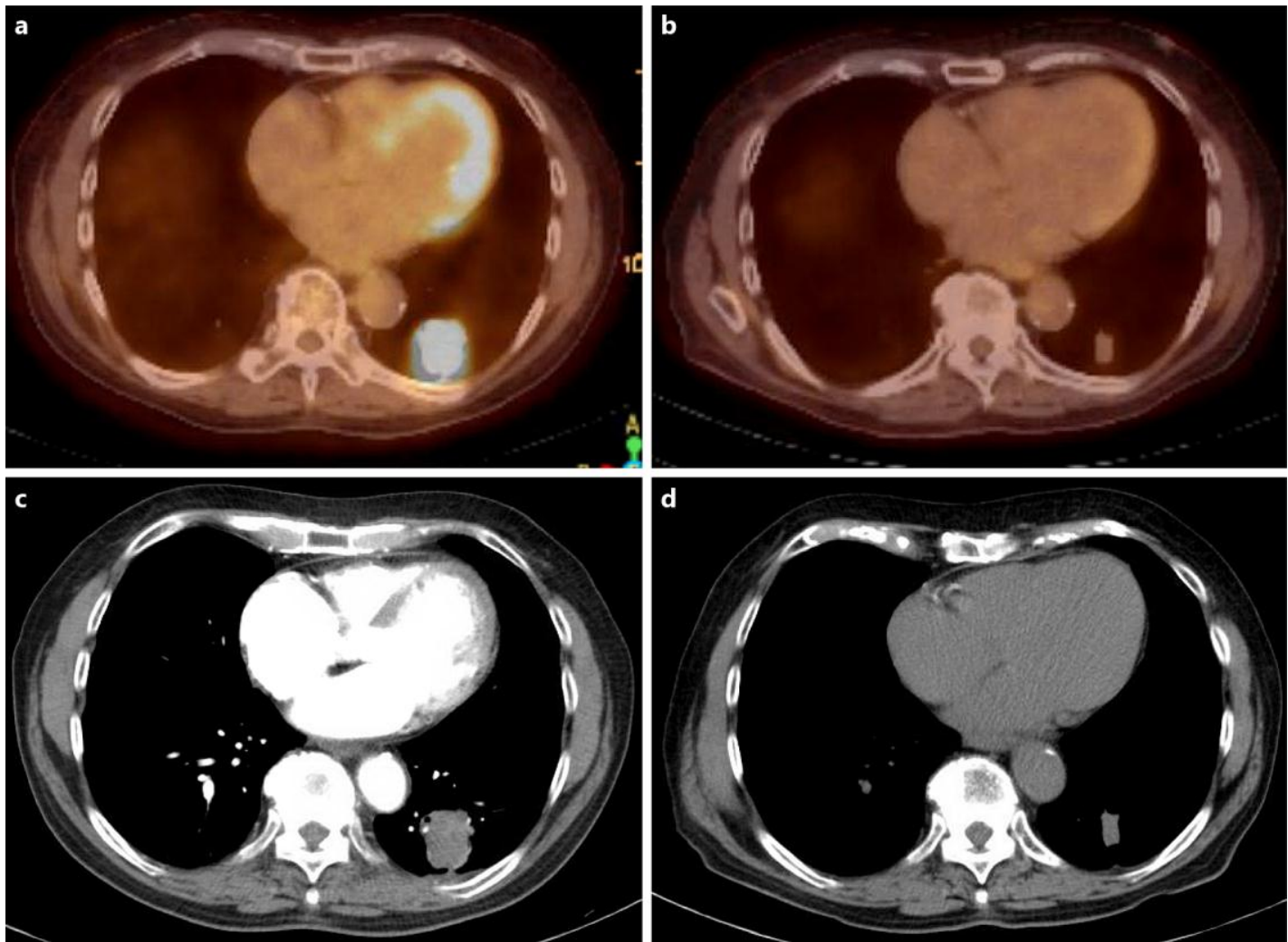

Fig. 1. Response to treatment. Primary tumor in the left lower lobe. Complete metabolic response to crizotinib as indicated by ${ }^{18}$ F-FDG-PET/CT scans before (a) and after 4.5 months of treatment (b). Partial response according to RECIST 1.1 criteria: tumor shrinkage from 33 to $13 \mathrm{~mm}$ before (c) and after 4.5 months of crizotinib (d). 


\section{Case Reports in Oncology}

\begin{tabular}{l|l}
\hline Case Rep Oncol 2016;9:158-163 \\
\hline DOI: $10.1159 / 000444745$ & $\begin{array}{l}\text { (c) 2016 The Author(s). Published by S. Karger AG, Basel } \\
\text { www.karger.com/cro }\end{array}$ \\
\hline
\end{tabular}

Overbeck et al.: Partial Response to First-Line Crizotinib in an Elderly Male Patient with ROS1 Translocation-Positive Lung Cancer
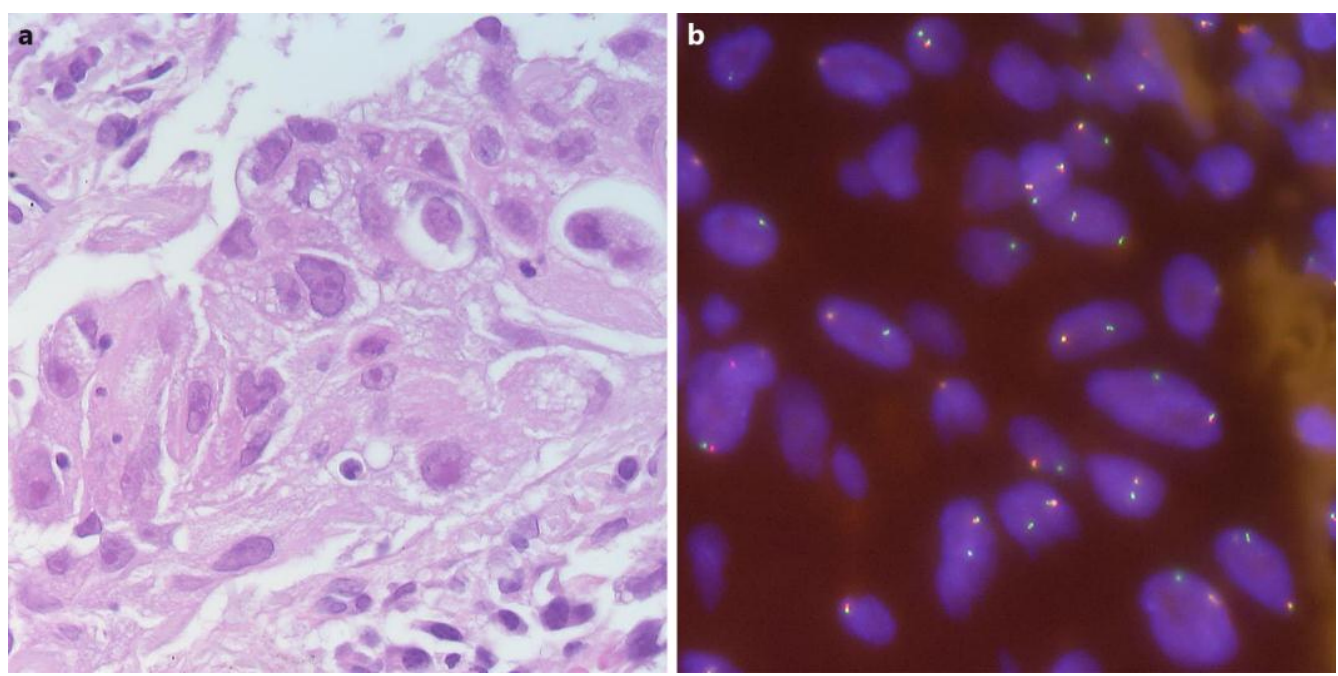

Fig. 2. Histological and molecular findings. a Solid type of pulmonary adenocarcinoma (H\&E). b ROS1 translocation detected by FISH. Green probes flank the 3' end of the ROS1 gene; normal cells display orange-green fusion signals. Gene rearrangements are indicated by either isolated green signals or breakapart signals of orange and green signals or a combination of both patterns in $\geq 15 \%$ of tumor cells (probe provided by ZytoVision, Bremerhaven, Germany). 